\title{
NOVOS FATORES DE CONVERSÃO DE CAROTENÓIDES PROVITAMÍNICOS A
}

\author{
Flávia Milagres CAMPOS ", Gilberto Paixão ROSADO
}

\section{RESUMO}

Este trabalho aborda os cálculos dos novos fatores de conversão de carotenóides provitamínicos A, propostos pelo Institute of Medicine, e agentes que afetam a biodisponibilidade de carotenóides em alimentos. Adicionalmente, revisa estudos que determinaram o conteúdo de carotenóides provitamínicos A em frutas e hortaliças no Brasil, para aplicar os novos fatores de conversão no cálculo do valor de vitamina A desses alimentos. O valor de vitamina A caiu pela metade ao se utilizarem os novos fatores, o que mostra que estes valores vêm sendo superestimados nas tabelas de composição química de alimentos. Paralelamente aos estudos demonstrando a baixa biodisponibilidade de carotenóides provenientes de vegetais, há estudos que apresentam o impacto positivo de vegetais e frutas ricos em carotenóides na concentração de retinol sérico. Medidas a curto e médio prazo, como a suplementação medicamentosa e a fortificação de alimentos, devem ser tomadas para conter a deficiência de vitamina A. A longo prazo, pode-se focalizar o consumo dos alimentos, como frutas e vegetais processados, que contêm carotenóides mais biodisponiveis. Enquanto os fatores que afetam a biodisponibilidade de carotenóides não estão bem definidos, as tabelas de composição devem trazer o conteúdo de carotenóides e não apenas o valor de vitamina A dos alimentos.

Palavras-chave: carotenos; vegetais; biodisponibilidade; vitamina A.

\section{SUMMARY}

NEW CONVERSION FACTORS OF PROVITAMIN A CAROTENOIDS. This work discusses how the new provitamin A carotenoids conversion factors proposed by the Institute of Medicine (IOM) were calculated and some of the factors that affect the bioavailability of carotenoids in foods. Additionally it reviews some studies that determined the carotenoid concentration of fruits and vegetables in Brazil, with the aim of applying the new conversion factors in the calculation of the vitamin A value of these foods. Vitamin A value dropped about $50 \%$ using the new conversion factors. This show that these values have been overestimated in food chemical composition tables. Concurrently to the studies that demonstrate that carotenoids from vegetables have low bioavailability, there are studies that show a positive impact of the carotenoid rich vegetables and fruits consumption in seric retinol. Short and median time actions, like supplementation and fortification of foodstuffs, should be taken in order to combat vitamin A deficiency. Long time actions could focus on the consumption of food in which carotenoids were more bioavailable like fruits and processed vegetables. While the factors that influence the bioavailability of carotenoids were not well defined, composition tables should bring carotenoid content instead of only vitamin A value.

Keywords: carotene; vegetables; bioavailability; vitamin A.

\section{1 - INTRODUÇÃO}

A deficiência de vitamina A continua sendo uma carência nutricional de impacto na saúde pública, pois atinge populações no mundo inteiro. Segundo a Organização Mundial da Saúde [48], só nas Américas a prevalência de hipovitaminose Aé de cerca de $20 \%$. Em grandes regiões brasileiras, constitui-se um problema endêmico [38]. Em revisão feita por GERALDO et al. [15], a deficiência de vitamina A foi detectada em vários estados brasileiros (Amazonas, Rio Grande do Norte, Paraíba, Pernambuco, Bahia, Minas Gerais, São Paulo e Santa Catarina), e, em alguns desses, a hipovitaminose A foi reconhecida como um problema de saúde pública. Entre as principais estratégias utilizadas no combate à deficiência de vitamina A nos países em desenvolvimento estão a suplementação medicamentosa, a fortificação de alimentos e mudanças na alimentação, incluindo maior consumo de vegetais ricos em carotenóides.

Recebido para publicação em 23/02/2005. Aceito para publicação em 28/07/2005(001491).

Departamento de Nutrição e Saúde, Universidade Federal de Viçosa. Campus Universitário, s/n. CEP:. 36571-000, Viçosa-MG, Brasil. Email:flaviamilagrescampos@yahoo.com.br / sepriore@mail.ufv.br A quem a correspondência deve ser enviada.
Entre as funções da vitamina A no organismo estão a participação no processo de visão, crescimento, diferenciação de tecidos, função imunológica, reprodução e desenvolvimento embrionário $[20,29]$. A carência de vitamina A pode levar à cegueira noturna, xeroftalmia, xerodermia e hiperqueratose folicular. Além disso, crianças com deficiência de vitamina A estão sob maior risco de sarampo, diarréia e infecções respiratórias [30, 40].

A vitamina A é um nutriente que se encontra apenas em alimentos de origem animal, em diversas formas: retinol, retinil, retinal e ácido retinóico. Os vegetais fornecem provitaminas $\mathrm{A}$, como o $\alpha$ e o $\beta$-caroteno e a $\beta$ criptoxantina, que podem ser biologicamente transformadas em vitamina A em organismos animais [34]. $O$ mais ativo dos carotenóides provitamínicos A é o $\beta$ caroteno [10], sendo também, o mais distribuído em alimentos, como o menor ou como o maior constituinte carotenogênico [35].

Populações em risco de deficiência de vitamina A, em geral, dependem de carotenóides provitamínicos A para atingirem suas recomendações diárias [33]. Segundo a Organização Mundial da Saúde [48], nas Américas, cerca de $64 \%$ da vitamina A ingerida é proveniente de carotenóides provitamínicos $\mathrm{A}$.

A composição de carotenóides em vegetais é afetada por diversos fatores como variedade, desigualdade na dis- 
tribuição em dado alimento e parte do vegetal que é consumido, grau de maturação, clima, tipo de solo, condições de cultivo e área geográfica de produção, condições de colheita, processamento e armazenamento [2, 19, 36].

Com clima tropical e subtropical, muitos países em desenvolvimento têm uma enorme variedade de fontes carotenogênicas [36], que incluem frutas, hortaliças e óleos extraídos de frutos de palmeiras diversas. Um exemplo disso é o óleo de dendê, que contém cerca de $16400 \mu \mathrm{g} / 100 \mathrm{~g}$ de RAE (Retinol Activity Equivalents)[14]. Esclarecer os fatores que afetam a biodisponibilidade de carotenóides em vegetais e aplicar fatores de conversão adequados para cálculo do valor de vitamina são medidas imprescindiveis para determinar até que ponto o consumo desses vegetais e óleos pode auxiliar no combate à deficiência de vitamina $A$.

Esta revisão aborda como foram calculados os novos fatores de conversão propostos pelo Institute of Medicine (IOM), discute alguns dos fatores que interferem na absorção de carotenóides e recalcula, com base nos novos fatores de conversão, o valor de vitamina A de algumas frutas e hortaliças comuns no Brasil, baseado no conteúdo de $\alpha$ e $\beta$-caroteno e $\beta$-criptoxantina dos vegetais reportados por estudos feitos em diferentes regiões do País.

\section{2 - FATORES DE CONVERSÃO}

Até a liberação das novas recomendações de nutrientes do Instituto Americano de Medicina (US Institute of Medicine - IOM), os fatores de conversão utilizados para cálculo do valor de vitamina A de um alimento de origem vegetal eram definidos como $1 \mu \mathrm{g}$ de retinol ou $12 \mu \mathrm{g}$ de $\alpha$-caroteno ou $6 \mu \mathrm{g}$ de $\beta$-caroteno correspondendo a $1 \mathrm{RE}$ (retinol equivalente) [27].

Estas equivalências, recomendadas também pelo Comitê de "Experts" da FAO/WHO, adotavam uma absorção intestinal de um terço do $\beta$-caroteno da dieta e uma eficiência de conversão de $50 \%$. Dessa forma, a utilização total do $\beta$-caroteno era tida como um sexto da utilização do retinol [34].

Em 2001, o IOM liberou as DRI's (Dietary Reference Intake) para vitamina A e outros nutrientes com recomendações nutricionais para a população americana e canadense [20]. Nesta publicação, o Instituto considerou novos fatores de conversão de carotenóides provitamini$\cos \mathrm{A}$, avaliando dois parâmetros: a eficiência de conversão de $\beta$-caroteno em retinol e sua taxa de absorção.

As novas recomendações mantêm o valor estimado de eficiência de conversão em $50 \%$, assim como a anterior [27]. A razão de equivalência $\beta$-caroteno: retinol foi mantida em 2:1 com base em estudo da década de 70 , que avaliou as quantidades de $\beta$-caroteno e retinol necessárias para corrigir anormalidades visuais em indivíduos que passaram por um período de depleção de vitamina A. É importante ressaltar que, neste estudo, o $\beta$ caroteno foi fornecido na forma de suplemento ( $\beta$ - caroteno puro em óleo). Portanto, cada $2 \mu \mathrm{g}$ de $\beta$-caroteno em óleo forneceram $1 \mu \mathrm{g}$ de retinol.

No entanto, este valor é contestado por WEST, EILANDER \& VAN LIESHOUT [47], que revisaram estudos que mostraram a necessidade de quantidades maiores de $\beta$-caroteno para fornecer $1 \mu \mathrm{g}$ de retinol. Alguns destes estudos foram ignorados pelo IOM, que alegou que estes usaram parâmetros não padronizados na avaliação das manifestações oculares da deficiência de vitamina A. Em estudo mais recente, utilizando isótopos, um dos autores chegou a um valor de conversão do $\beta$ caroteno em óleo bem próximo de $2 \mu \mathrm{g}$ de $\beta$-caroteno para formar $1 \mu \mathrm{g}$ de retinol [46].

Considera-se que uma vez absorvido, o $\beta$-caroteno proveniente de uma dieta mista ou de suplemento segue as mesmas rotas metabólicas, o que justifica a utilização de $50 \%$ como fator de eficiência de conversão de $\beta$ caroteno para retinol tanto para $\beta$-caroteno proveniente de uma dieta mista quanto para o proveniente de suplemento.

Entretanto, a absorção de carotenóides concentrados em óleo é maior que a absorção de carotenóides presentes em alimentos e em combinações de alimentos numa dieta mista. Portanto, as taxas de absorção de $\beta$ caroteno de diferentes alimentos em relação à absorção do caroteno em óleo responderam pelas alterações nos fatores de conversão. Ao invés dos valores de absorção de $33 \%$ do $\beta$-caroteno em uma dieta mista em relação ao $\beta$ caroteno em óleo, o Instituto de Medicina preferiu considerar uma taxa de absorção de $14 \%$, ou seja, cerca da metade do que se acreditava anteriormente.

Estes valores são baseados em um estudo de VAN het HOLF et al. [44], em que, durante quatro semanas, 54 indivíduos divididos em três grupos consumiram uma dieta rica em vegetais, uma dieta pobre em vegetais e uma dieta pobre em vegetais suplementada com $\beta$ caroteno. $\mathrm{O} \beta$-caroteno sérico foi utilizado como parâmetro para avaliar a absorção da provitamina, que mostrou ser de $14 \%$. Além disso, outros estudos que analisaram a absorção de $\beta$-caroteno proveniente de vegetais isolados ou de grupos do mesmo tipo de vegetais foram utilizados para dar suporte a este valor de absorção relativa de $14 \%$. Nestes trabalhos, a absorção de $\beta$-caroteno esteve entre 18 e $26 \%$ para cenoura e entre 4 e $12 \%$ para vegetais folhosos [9, 11, 12, 25, 43]. Embora uma absorção de $14 \%$ corresponda a uma relação $\beta$-caroteno da dieta: $\beta$ caroteno em óleo igual a 7:1, o IOM preferiu utilizar o valor de $6: 1$, justificado pelo fato de que, no estudo de VAN het $\mathrm{HOF}$ et al. [44], os carotenóides da dieta provinham mais de legumes do que de frutas, e estes, por sua vez, têm uma absorção maior do que os carotenóides em legumes e vegetais folhosos, como foi demonstrado por de $\mathrm{PEE}$ et al. [12]. Segundo VAN het HOLF et al. [45], a diferença entre a absorção de carotenóides de frutas e legumes pode ser devida a diferenças na localização intrace- 
lular dos carotenóides.

Dessa forma, os novos fatores de conversão de carotenóides provitamínicos estabelecidos pelo IOM são o dobro dos fatores utilizados até a última recomendação. O cálculo foi feito da seguinte forma: $2: 1$ × $6: 1=12: 1$, isto é, 2: 1 corresponde à taxa de conversão de $\beta$-caroteno (cada $2 \mu \mathrm{g}$ de $\beta$-caroteno em óleo correspondem a $1 \mu \mathrm{g}$ de retinol), enquanto $6: 1$ corresponde à absorção de $\beta$-caroteno de uma dieta mista de vegetais em relação à absorção de $\beta$-caroteno puro em óleo (aproximadamente 14\%).

Neste ponto, o IOM introduz um novo conceito, o de Equivalente de Atividade de Retinol (RAE). Cada Equivalente de Atividade de Retinol corresponde a $1 \mu \mathrm{g}$ de retinol ou $12 \mu \mathrm{g}$ de $\beta$-caroteno ou $24 \mu \mathrm{g}$ de outros carotenóides provitamínicos. O fator de conversão de outros carotenóides provitamínicos foi estabelecido por extrapolação, com base no fato de que sua atividade provitamínica A é considerada cerca de metade da atividade do $\beta$ caroteno.

Há divergência na literatura quanto à biodisponibilidade e à eficácia de carotenóides provitamínicos A no combate à deficiência da vitamina. Alguns estudos mostram que o consumo de vegetais ricos em carotenóides pode melhorar a concentração sérica de retinol ou manter um bom estado nutricional de vitamina A [21, 28, 32, 42]. Já em outros estudos, os vegetais ricos em carotenóides mostraram pouco ou nenhum efeito na elevação da concentração sérica de retinol [11, 12, 25].

Discutindo estas divergências dos resultados, os autores relatam que, nos estudos em que o consumo de vegetais ricos em carotenóides não aumentou a concentração sérica de retinol, os indivíduos tinham bons estoques de vitamina A antes do início dos experimentos, o que teria influenciado os resultados $[21,28,32]$.

WEST, EILANDER \& VAN LIESHOUT [47] apontam para o fato de que os novos fatores de conversão utilizados pelo IOM ainda podem estar superestimando o valor de vitamina A de alimentos de origem vegetal, uma vez que a biodisponibilidade de carotenóides de alguns tipos de vegetais pode ser ainda mais baixa.

No estudo de TANG et al. [42], embora tenha sido demonstrada a influência positiva dos vegetais verdes e amarelos na manutenção da concentração sérica de retinol, ao estimar a quantidade de $\beta$-caroteno da dieta necessária para produzir $1 \mu \mathrm{g}$ de retinol, encontrou-se um valor de 27:1. de PEE et al. [12] encontraram fatores de conversão de $\beta$-caroteno de 12: 1 em frutas e 26: 1 em legumes.

\section{3 - FATORES QUE AFETAM A ABSORÇÃO}

Os fatores que afetam a absorção de carotenóides são: tipo de carotenóide ingerido, ligações moleculares, quantidade de carotenóide na dieta, matriz em que o carotenóide se encontra, presença de fatores inibidores ou facilitadores da absorção, estado nutricional do indivíduo, fatores genéticos, fatores relacionados com o indivíduo e interação entre estas variáveis [45].

Dentre os fatores que influenciam na biodisponibilidade de carotenóides, os relacionados à dieta têm sido os mais intensamente estudados. O principal é o tipo de alimento em que eles estão presentes [33, 45, 47]. Isto se deve ao fato de que os carotenóides estão situados em locais diferentes numa mesma planta ou em plantas diversas. Os carotenóides são encontrados no complexo pigmentoproteína nos cloroplastos de tecidos verdes, onde sua cor é mascarada pelas clorofilas, e em tecidos nãofotossintéticos, na forma cristalina nos cromoplastos, onde são responsáveis pela cor de muitas flores e frutos [5]. Além disso, a presença de fibras pode também interferir na biodisponibilidade de carotenóides [45].

O processamento e a homogeneização mecânica dos alimentos e a conseqüente redução do tamanho das partículas podem aumentar a biodisponibilidade dos carotenóides [13, 28, 45]. Alguns estudos que analisaram o conteúdo de carotenóides em alimentos, reportam maior facilidade de extração dos carotenóides, após o processamento térmico, produzindo aumento na quantidade total de carotenóides provitamínicos A em relação aos vegetais frescos [18, 22, 23]. Isto se deve provavelmente a uma desnaturação mais eficiente dos complexos carotenóideproteína [22]. VAN het HOLF et al. [45] relatam que esta extração aumentada pode estar associada também à maior biodisponibilidade de carotenóides provenientes de matriz vegetal submetida a tratamento térmico.

ROCK et al.[33] encontraram aumento na biodisponibilidade de $\beta$-caroteno em vegetais processados. O processamento, como o tratamento térmico tem o potencial de elevar a biodisponibilidade de carotenóides provenientes de vegetais.

No entanto, deve-se ressaltar que o tratamento térmico promove a isomerização dos carotenóides nos alimentos, da forma isomérica trans para cis, e o grau de isomerização está diretamente relacionado com a intensidade e a duração do tratamento térmico [33]. Nutricionalmente, a diferenciação entre os isômeros cis e trans das provitaminas é importante, porque a forma cis exibe menor potência [34].

Um estudo mostrou que a destruição da matriz do espinafre por tratamento enzimático (celulases e pectinases) aumentou a resposta plasmática do $\beta$-caroteno em cerca de $65 \%$ em homens e mulheres com idade entre 18 e 58 anos [9]. A resposta plasmática à ingestão de carotenóides foi maior em mulheres adultas quando utilizado suco de cenoura do que cenoura [43]; e a absorção de $\alpha$ e $\beta$-caroteno foi cerca de duas vezes maior na cenoura em forma de purê do que na cenoura cozida [13], mostrando que a destruição da matriz aumenta a biodisponibilidade dos carotenóides.

O consumo de lipídios, paralelo ao consumo de carotenóides, parece ser de extrema importância para a absorção dos últimos [45]. Uma vez que os carotenóides são 
lipossolúveis e são absorvidos, em geral, de maneira semelhante aos demais lipídios da dieta, eles necessitam da formação de micelas na luz intestinal para garantir sua solubilização, e a presença de fatores que interferem na formação das micelas podem influenciar na absorção dos carotenóides. Em estudo feito por JALAL et al. [21], houve aumento no retinol sérico em crianças de 3 a 6 anos de idade com o acréscimo de 3 e de $5 \mathrm{~g}$ de lipídios em um produto à base de batata-doce, ou seja, pequenas quantidades de lipídios são suficientes para garantir a absorção dos carotenóides.

Além desses fatores relacionados diretamente à dieta, observa-se também a interferência de parasitas intestinais na absorção dos carotenóides da dieta [21] e do es- tado nutricional de vitamina A na conversão das provitaminas em retinol. Segundo RIBAYA-MERCADO et al. [32], quanto maiores os estoques de vitamina $A$, menor é a taxa de conversão dos carotenóides.

\section{4 - CÁlCULOS DO VALOR DE VITAMINA A COM BASE NOS NOVOS FATORES DE CONVERSÃO}

Com os novos fatores de conversão utilizados pelo Instituto de Medicina (IOM), o valor provitamínico A de vegetais passa a ser a metade dos valores que vinham sendo utilizados até então. A quantidade de vitamina A em $\mu \mathrm{g}$ RAE é metade da quantidade em $\mu \mathrm{g}$ RE, o que mostra que os valores de provitaminas A vêm sendo superesti-

TABELA 1 - Conteúdo de $\alpha$ e $\beta$-caroteno, $\beta$-criptoxantina ( $\mu$ g/ 100g) e valor de vitamina A ( $\mu$ g/ $100 g$ RAE) de vegetais folhosos

\begin{tabular}{|c|c|c|c|c|c|c|c|}
\hline Referência & Vegetal (1) & Preparo (2) & $\alpha$-caroteno & $\beta$-caroteno & $\beta$-criptoxantina & $\begin{array}{c}\text { Valor de } \\
\text { vitamina A }\end{array}$ & Local (3) \\
\hline [6] & agriāo & cru & -- & 4330 & $=$ & 361 & Viçosa, MG \\
\hline \multirow[t]{2}{*}[8]{$^{(5)}$} & agriāo & fresco & 857 & 8631 & $\ldots$ & 755 & Viçosa, MG \\
\hline & agrião hidropônico & fresco & 735 & 8751 & $\cdots$ & 760 & Viçosa, MG \\
\hline [6] & alface & cru & ... & 1482 & $\ldots$ & 124 & Viçosa, MG \\
\hline$[8]^{(5)}$ & alface crespa & fresca & 198 & 2602 & $\ldots$ & 225 & Viçosa, MG \\
\hline$[8]^{(5)}$ & alface lisa & fresca & 191 & 2857 & $\cdots$ & 238 & Viçosa, MG \\
\hline [4] & bertalha & fresca & $\cdots$ & 2841 & $-\cdot$ & 237 & Viçosa, MG \\
\hline [26] & brócolis & fresco & $\ldots$ & 2113 & $\ldots$ & 194 & Viçosa, MG \\
\hline \multirow{2}{*}{ [37] } & brócolis & cozido & ... & $1893^{(4)}$ & -.. & 158 & Campinas, SP \\
\hline & brócolis & refogado & $\cdots$ & $1575^{(4)}$ & -.. & 131 & Campinas, SP \\
\hline \multirow{2}{*}{ [6] } & brócolis & fresco & $\ldots$ & 2323 & -.. & 194 & Viçosa, MG \\
\hline & brócolis & cozido & ... & 2100 & $\ldots$ & 175 & Viçosa, MG \\
\hline \multirow[t]{2}{*}{ [17] } & brócolis & fresco & ... & 1810 & ... & 151 & Campinas, SP \\
\hline & brócolis & cozido & $\cdots$ & 1490 & $\ldots$ & 124 & Campinas, SP \\
\hline [26] & cebolinha & fresca & -.- & 2944 & $\ldots$ & 245 & Viçosa, MG \\
\hline [4] & cebolinha & fresca & -. & 2273 & -.- & 189 & Viçosa, MG \\
\hline [6] & cebolinha & crua & $\cdots$ & 2220 & $\cdots$ & 185 & Viçosa, MG \\
\hline [26] & chicória & fresca & $\cdots$ & 4179 & $\cdots$ & 348 & Viçosa, MG \\
\hline [4] & coentro & fresco & $\cdots$ & 5763 & $\cdots$ & 480 & Viçosa, MG \\
\hline [37] & couve & refogada & -.. & $2307^{(4)}$ & $\ldots$ & 192 & Campinas, SP \\
\hline$[8]^{(5)}$ & couve & fresca & 448 & 8423 & $\ldots$ & 721 & Viçosa, MG \\
\hline$[17]$ & couve & fresca & -.. & 2580 & -.. & 215 & Campinas, SP \\
\hline [26] & couve-chinesa & fresca & ... & 2432 & $\ldots$ & 203 & Viçosa, MG \\
\hline$[8]$ (i) & espinafre & fresco & 297 & 5726 & $\cdots$ & 490 & Viçosa, MG \\
\hline [17] & espinafre & fresco & $\cdots$ & 2500 & -. & 208 & Campinas, SP \\
\hline$[17]$ & espinafre & cozido & $\cdots$ & 2050 & $\cdots$ & 171 & Campinas, SP \\
\hline [26] & hortelã & fresca & --. & 4997 & -- & 416 & Viçosa, MG \\
\hline [4] & manjericão & fresco & $\cdots$ & 3899 & $\cdots$ & 325 & Viçosa, MG \\
\hline [26] & mostarda & fresca & $\cdots$ & 3786 & $\cdots$ & 316 & Viçosa, MG \\
\hline [26] & rúcula & fresca & $\cdots$ & 4865 & -- & 405 & Viçosa, MG \\
\hline [26] & hortelã & fresca & $\cdots$ & 4997 & $\cdots$ & 416 & Viçosa, MG \\
\hline [4] & manjericão & fresco & ... & 3899 & -.. & 325 & Viçosa, MG \\
\hline [26] & mostarda & fresca & $\cdots$ & 3786 & $\cdots$ & 316 & Viçosa, MG \\
\hline [26] & rúcula & fresca & -.- & 4865 & -- & 405 & Viçosa, MG \\
\hline [26] & salsa & fresca & $\cdots$ & 6357 & $\ldots$ & 530 & Viçosa, MG \\
\hline [4] & salsa & fresca & -.. & 6233 & -.. & 519 & Viçosa, MG \\
\hline [6] & salsa & crua & $\cdots$ & 4203 & $\cdots$ & 350 & Viçosa, MG \\
\hline [26] & salsăo & fresca & $\cdots$ & 1999 & $\cdots$ & 167 & Viçosa, MG \\
\hline [26] & serralha & fresca & -.- & 6872 & -- & 573 & Viçosa, MG \\
\hline$[26]$ & taioba & fresca & $\ldots$ & 7261 & $\ldots$ & 605 & Viçosa, MG \\
\hline
\end{tabular}


Fatores de conversão carotenóides, Campos \& Rosado

TABELA 2 - Conteúdo de $\alpha$ e $\beta$-caroteno, $\beta$-criptoxantina ( $\mu$ g/ 100g) e valor de vitamina A ( $\mu$ g/ 100g RAE) de hortaliças

\begin{tabular}{|c|c|c|c|c|c|c|c|}
\hline Referência & Vegetal (1) & Preparo & $\alpha$-caroteno & $\beta$-caroteno & $\beta$-criptoxantina & $\begin{array}{c}\text { Valor de } \\
\text { vitamina A }\end{array}$ & Local (2) \\
\hline [26] & abobrinha & crua & $\ldots$ & 154 & $\ldots$ & 13 & Viçosa, MG \\
\hline$[7]^{[3]}$ & cenoura & fresca & 3110 & 5950 & -.. & 625 & Viçosa, MG \\
\hline \multirow[t]{4}{*}[17]{} & cenoura (Imperador) & fresca & 1900 & 3840 & --. & 399 & Campinas, SP \\
\hline & cenoura (Imperador) & cozida & 1630 & 3610 & ... & 369 & Campinas, SP \\
\hline & cenoura (Nantes) & fresca & 1650 & 3300 & $\ldots$ & 344 & Campinas, SP \\
\hline & cenoura (Nantes) & cozida & 1340 & 3030 & $\ldots$ & 308 & Campinas, SP \\
\hline [31] & cenoura (Nantes) & cozida & 3414 & 7275 & $\cdots$ & 749 & Viçosa, MG \\
\hline$[7]^{(3)}$ & moranga & fresca & 436 & 2318 & ... & 211 & Viçosa, MG \\
\hline \multirow[t]{3}{*}{ [17] } & moranga & fresca & 1710 & 2360 & -.. & 268 & Campinas, SP \\
\hline & moranga & cozida & 1560 & 2110 & -.. & 241 & Campinas, SP \\
\hline & moranga & refogada & 1800 & 2070 & $\cdots$ & 248 & Campinas, SP \\
\hline$[7]^{(3)}$ & pimentão verde & fresco & 048 & 453 & ... & 40 & Viçosa, MG \\
\hline [17] & pimentão verde & fresco & & 210 & $\cdots$ & 18 & Campinas, $\mathrm{SP}$ \\
\hline$[7]^{(3)}$ & quiabo & fresco & 33 & 391 & $\ldots$ & 34 & Viçosa, MG \\
\hline \multirow[t]{2}{*}{ [17] } & quiabo & fresco & ... & 270 & ... & 23 & Campinas, SP \\
\hline & quiabo & cozido & ... & 180 & ... & 15 & Campinas, $\mathrm{SP}$ \\
\hline$[7]^{(3)}$ & tomate & fresco & ... & 599 & ... & 50 & Viçosa, MG \\
\hline \multirow[t]{2}{*}[17]{} & tomate (Sta. cruz) & fresco & ... & 510 & ... & 43 & Campinas, SP \\
\hline & tomate (Marglobe) & fresco & -.. & 620 & -.. & 52 & Campinas, SP \\
\hline \multirow[t]{2}{*}{ [37] } & vagem & cozida & -.. & 163 & -.. & 14 & Campinas, SP \\
\hline & vagem & refogada & -.. & 176 & -.. & 15 & Campinas, SP \\
\hline$[7]^{(3)}$ & vagem & fresca & 80 & 420 & -.. & 38 & Viçosa, MG \\
\hline \multirow{3}{*}{ [17] } & vagem & fresca & 20 & 120 & ... & 11 & Campinas, SP \\
\hline & vagem & cozida & 20 & 100 & -.. & 09 & Campinas, SP \\
\hline & vagem & refogada & ... & 80 & $\ldots$ & 07 & Campinas, SP \\
\hline
\end{tabular}

proveniência dos vegetais. [i] Média de très estaçōes e dois pontos de coleta.

TABELA 3 - Conteúdo de $\alpha$ e $\beta$-caroteno, $\beta$-criptoxantina ( $\mu \mathrm{g} / 100 \mathrm{~g}$ ) e valor de vitamina A ( $\mu \mathrm{g} / 100 \mathrm{~g}$ RAE) de frutas

\begin{tabular}{|c|c|c|c|c|c|c|c|}
\hline Referência & Vegetal & Preparo & $\alpha$-caroteno & $\beta$-caroteno & $\beta$-criptoxantina & $\begin{array}{c}\text { Valor de } \\
\text { Vitamina A }\end{array}$ & Local \\
\hline $\begin{array}{l}1] \\
{[41]}\end{array}$ & $\begin{array}{l}\text { acerola } \\
\text { acerola }\end{array}$ & $\begin{array}{l}\text { polpa } \\
\text { fresca }\end{array}$ & 20 & $\begin{array}{r}710 \\
1041\end{array}$ & $\begin{array}{l}170 \\
---\end{array}$ & $\begin{array}{l}67 \\
87\end{array}$ & $\begin{array}{l}\text { Fortaleza, CE } \\
\text { Viçosa, MG }\end{array}$ \\
\hline [41] & ameixa & fresca & $\cdots$ & 257 & $\cdots$ & 21 & Viçosa, MG \\
\hline [16] & buriti & fresco & 8010 & 35980 & -.. & 3332 & Piauí \\
\hline [16] & cajá & fresco & 30 & 140 & 1690 & 83 & $\begin{array}{l}\text { Rio Grande do } \\
\text { Norte }\end{array}$ \\
\hline [41] & caqui & fresca & $\cdots$ & 352 & $\cdots$ & 29 & Viçosa, MG \\
\hline [41] & carambola & fresca & 41 & 85 & $\cdots$ & 09 & Viçosa, MG \\
\hline [3] (3) & goiaba & fresca & $\cdots$ & 1050 & $\cdots$ & 88 & Viçosa, MG \\
\hline$[3]^{(3)}$ & kiwi & fresco & -- & 190 & $\cdots$ & 16 & Viçosa, MG \\
\hline $\begin{array}{l}{[39]} \\
{[16]} \\
{[3]^{(3)}} \\
{[41]}\end{array}$ & $\begin{array}{l}\text { maracujá } \\
\text { maracujá } \\
\text { maracujấ } \\
\text { maracujá doce }\end{array}$ & $\begin{array}{l}\text { fresco } \\
\text { fresco } \\
\text { fresco } \\
\text { fresco }\end{array}$ & $\begin{array}{l}\cdots \\
\cdots \\
\cdots\end{array}$ & $\begin{array}{c}762 \\
470 \\
1960 \\
559\end{array}$ & $\begin{array}{c}150 \\
--- \\
--- \\
--\end{array}$ & $\begin{array}{c}70 \\
39 \\
163 \\
47\end{array}$ & $\begin{array}{l}\text { Campinas, SP } \\
\text { São Paulo } \\
\text { Viçosa, MG } \\
\text { Viçosa, MG }\end{array}$ \\
\hline $\begin{array}{l}{[24]} \\
{[16]} \\
{[3]^{13]}}\end{array}$ & $\begin{array}{l}\text { manga } \\
\text { manga (cv Haden) } \\
\text { manga (cv Atkins) } \\
\text { manga }\end{array}$ & $\begin{array}{l}\text { fresca } \\
\text { fresca } \\
\text { fresca } \\
\text { fresca }\end{array}$ & $\cdots$ & $\begin{array}{l}1510 \\
1250 \\
1550 \\
1480\end{array}$ & $\begin{array}{l}30 \\
-- \\
\cdots- \\
--\end{array}$ & $\begin{array}{l}127 \\
104 \\
129 \\
123\end{array}$ & $\begin{array}{l}\text { Campinas, SP } \\
\text { São Paulo } \\
\text { Mato Grosso } \\
\text { Viçosa, MG }\end{array}$ \\
\hline $\begin{array}{l}{[16]} \\
{[16]}\end{array}$ & $\begin{array}{l}\text { mamão (cv Solo) } \\
\text { mamâo (cv } \\
\text { Tailândia) }\end{array}$ & $\begin{array}{l}\text { fresco } \\
\text { fresco }\end{array}$ & $\cdots$ & $\begin{array}{l}300 \\
260\end{array}$ & $\begin{array}{c}760 \\
1020\end{array}$ & $\begin{array}{l}57 \\
64\end{array}$ & $\begin{array}{l}\text { São Paulo } \\
\text { Bahia }\end{array}$ \\
\hline $\begin{array}{l}{[3]^{(3)}} \\
{[41]}\end{array}$ & $\begin{array}{l}\text { mamão-papaya } \\
\text { mamâo-formosa }\end{array}$ & $\begin{array}{l}\text { fresco } \\
\text { fresco }\end{array}$ & 3,38 & $\begin{array}{l}370 \\
280\end{array}$ & $\cdots$ & $\begin{array}{l}31 \\
37\end{array}$ & $\begin{array}{l}\text { Viçosa, MG } \\
\text { Viçosa, MG }\end{array}$ \\
\hline [41] & morango & fresco & $\cdots$ & 21 & $\cdots$ & 02 & Viçosa, MG \\
\hline$\left[\begin{array}{l}41] \\
{[16]}\end{array}\right.$ & $\begin{array}{l}\text { nectarina } \\
\text { nectarina }\end{array}$ & $\begin{array}{l}\text { fresca } \\
\text { fresca }\end{array}$ & $\cdots$ & $\begin{array}{l}154 \\
110\end{array}$ & 390 & $\begin{array}{l}13 \\
25\end{array}$ & $\begin{array}{l}\text { Viçosa, MG } \\
\text { São Paulo }\end{array}$ \\
\hline $\begin{array}{l}{[41]} \\
{[16]}\end{array}$ & $\begin{array}{l}\text { nêspera } \\
\text { nêspera }\end{array}$ & $\begin{array}{l}\text { fresca } \\
\text { fresca }\end{array}$ & $\cdots$ & $\begin{array}{c}2069 \\
800\end{array}$ & 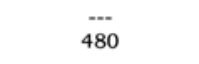 & $\begin{array}{l}172 \\
87\end{array}$ & $\begin{array}{l}\text { Viçosa, MG } \\
\text { São Paulo }\end{array}$ \\
\hline [16] & pêra (cv radiante) & fresca & $\cdots$ & 60 & 410 & 22 & São Paulo \\
\hline [16] & pequi & fresco & 0,10 & 120 & 440 & 29 & Piauí \\
\hline [16] & pitanga & fresca & $\ldots$ & 370 & 1230 & 82 & Campinas, SP \\
\hline
\end{tabular}

náo-detectado ou não-analisado. (1) Nos estudos em que

proveniència dos vegetais. ${ }^{(3)}$ Resultados obtidos durante a primavera. 
mados nas tabelas de composição química de alimentos.

Estas tabelas são primordiais no desenvolvimento do trabalho dos nutricionistas, uma vez que são utilizadas na elaboração de dietas individuais durante o atendimento ambulatorial, na elaboração de cardápios nutricionalmente balanceados para coletividades em Unidades de Alimentação e Nutrição e na análise de estudos da ingestão de nutrientes e adequação nutricional de populações. Portanto, é necessário chamar a atenção destes profissionais para a forma como o valor de vitamina $\mathrm{A}$ de alimentos de origem vegetal é expressa nestas tabelas.

As Tabelas 1, 2 e 3 trazem o conteúdo de $\alpha$ e $\beta$ caroteno, $\beta$-criptoxantina e o valor de vitamina A segundo os novos fatores de conversão de vegetais folhosos, legumes e frutas analisados em estudos no Brasil. O $\alpha$ e o $\beta$-caroteno e a $\beta$-criptoxantina podem ser biologicamente transformados em vitamina A em organismos animais [34], constituindo-se nos principais carotenóides provitaminicos A largamente distribuídos em alimentos.

Ressalta-se, mais uma vez, que a variedade do vegetal, o grau de maturação, as condições e área de cultivo podem influenciar o conteúdo de carotenóides de vegetais [36], além do processamento térmico.

Em alguns estudos foi feita a separação dos isômeros cis e trans de $\alpha$ e $\beta$-caroteno e $\beta$-criptoxantina e em outros não. Isso pode ter causado uma superestimação do valor de vitamina A. Um dos estudos mostrou que, em frutas frescas, a não separação dos isômeros resultou em uma superestimação do valor de vitamina A entre 3 e $10 \%$, o que é uma porcentagem pequena [16]. Neste trabalho, foi considerado apenas o valor das formas trans, uma vez que estas têm significância maior.

Mesmo nos estudos em que foi feita a determinação de umidade dos vegetais, os valores são apresentados na base úmida nas Tabelas 1, 2 e 3 .

\section{5 - CONSIDERAÇÕES FINAIS}

Os novos fatores de conversão propostos pelo IOM dificultam muito a proposta de atingir as recomendações de vitamina A com o consumo de vegetais ricos em carotenóides provitamínicos, o que pode ter sérias implicações nas estratégias de combate à hipovitaminose $\mathrm{A}$.

Embora boa parte dos estudos tenha repetidamente mostrado uma baixa absorção dos carotenóides provenientes de vegetais, outros estudos têm demonstrado o impacto positivo do consumo de vegetais e frutas ricos em $\beta$-caroteno na concentração de retinol sérico. A baixa biodisponibilidade dos carotenóides da dieta pode interferir na eficácia do uso destes no combate à hipovitaminose A. Contudo, o consumo de vegetais fontes de carotenóides provitamínicos poderia manter o estado nutricional adequado de vitamina A em indivíduos normais.

Parece que deixar de lado esta estratégia não é a melhor opção, especialmente em países em desenvolvimento. Educação nutricional deve ser o norteador das ações a longo prazo. As ações devem focalizar o consumo dos alimentos em que os carotenóides têm sido apontados como mais disponiveis, como as frutas, e as formas de aumentar a biodisponibilidade de carotenóides, como o consumo de vegetais processados. Além disso, o Brasil possui uma enorme variedade de frutos cujo óleo rico em carotenóides de boa biodisponibilidade pode ser extraído e utilizado na alimentação.

Apesar disso, não é possivel ignorar o fato de que com os novos fatores de conversão é muito difícil atingir a recomendação de vitamina A apenas com o consumo de vegetais fontes de carotenóides provitamínicos, visto que o valor de vitamina A dos vegetais caiu pela metade. Em locais onde a deficiência se torna um problema de saúde pública, outras medidas devem ser tomadas para conter a deficiência a curto prazo, como a suplementação com megadoses de vitamina A aos grupos de risco, assim como vem sendo realizado pelo Ministério da Saúde no Brasil. Além disso, a fortificação de alimentos industrializados também pode ser utilizada para melhorar o suprimento de vitamina A para a população em geral.

Salienta-se que, até o presente, as tabelas de composição química de alimentos trazem os valores de vitamina A de alimentos de origem vegetal na forma de Retinol Equivalente (RE). Portanto, é preciso estar atento ao se lidar com estas tabelas. A melhor opção seria que estas trouxessem o conteúdo de carotenóides e não apenas o valor de vitamina A dos alimentos, especialmente enquanto os fatores que afetam a biodisponibilidade de carotenóides não estão bem definidos. Provavelmente, no futuro, haja fatores de conversão diferentes de acordo com a matriz em que o carotenóide se encontra. Enquanto isso, é necessário ter prudência ao utilizar o valor de vitamina A de alimentos de origem vegetal.

\section{6 - REFERÊNCIAS BIBLIOGRÁFICAS}

[1] Agostini-Costa, T. S.; ABREU, L. N.; ROSSETTI, A. Efeito do congelamento e do tempo de estocagem da polpa de acerola sobre o teor de carotenóides. Rev. Bras. Frutic., v. 25, n. 1, p. 56-58, 2003.

[2] AMAYA-FARFAN, J. Panorama de la investigación sobre carotenoides en el Brasil. Perspectiva y necesidades. Arch. Latinoam. Nutr., v. 49, p. 92-94, 1999.

[3] AMORIM, N.M.L.; SANT'ANA, H.M.P.; STRINGHETA, P.C.; CHAVES, J.B.P.; CARDOSO, P.C.; MOREIRA, A.P.B.; SOUZA, S.L.. Avaliação de Alfa e Beta-Caroteno em Frutas Comercializadas em Viçosa-MG. In: XII Simpósio de Iniciação Científica da UFV. Desafio Brasil: Prioridade na Pesquisa e Desenvolvimento. Editora da UFV, 2002. v. 1, p. 214-215.

[4] BATISTA, M.A. \& PINHEIRO-SANT'ANA, H.M. Avaliação de carotenóides em vegetais comercializados em Viçosa-MG. Relatório Final apresentado à Universidade Federal de Viçosa, como parte das exigências do PIBIC/CNPq. 2004. 50p.

[5] BRITTON, G. Carotenoids. In: CHARLWOOD, B. \& BANTHORPE, D. eds. Methods in plant biochemistry, London, Academy Press, 1991.v. 7. p. 473-518. 
[6] CAMPOS, F. M., PINHEIRO-SANT ANA, H. M., STRINGHETA, P. C., CHAVES, J. B. P. Teores de beta caroteno em vegetais folhosos preparados em restaurantes comerciais de Viçosa-MG. Braz. J. Food Technol.. Campinas, v. 6, n. 2, p.163 - 169, 2003.

[7] CAMPOS, F. M.; PINHEIRO-SANT'ANA, H. M.; SOUZA, P. M.; STRINGHETA, P. C., CHAVES; J. B. P. Avaliação de alfa e beta caroteno em vegetais comercializados na cidade de Viçosa-MG em três estações do ano In: VI Congresso da Sociedade Brasileira de Alimentação e Nutrição (SBAN) - Nutrição e Alimentação: da Adequação à Excelência, Florianópolis, SC, 2001. v. 1. p. 317317.

[8] CARDOSO, P.C.; SANT'ANA, H.M.P.; STRINGHETA, P.C.; CHAVES, J.B.P.; AMORIM, N.M.L.; MOREIRA, A.P.B.; SOUZA, S.L.. Avaliação de Carotenóides em Vegetais Folhosos Comercializados em Viçosa-MG. In: XII Simpósio de Iniciação Científica da UFV. Desafio Brasil: Prioridade na Pesquisa e Desenvolvimento. Viçosa: Editora UFV, 2002.v. 1, p. 215-215.

[9] CASTENMILLER, J. J. M; WEST, C. E.; LINSSEN, J. P. H.; VAN HET HOF, K. H.; VORAGEN, A. G. J. The Food Matrix of Spinach Is a Limiting Factor in Determining the Bioavailability of $\beta$-Carotene and to a Lesser Extent of Lutein in Humans. J. Nutr., v. 129, p. 349355, 1999.

[10] COMBS JUNIOR, G.F. Vitaminas. In: Mahan, L.K. \& Escott-Stump, S. (eds.). Krause: alimentos, nutrição \& dietoterapia. São Paulo, Roca, 2002 . p. 65-105.

[11] de PEE, S.; WEST C. E.; MUHILAL, KARYADI D.; HAUTVAST J.G.A.J. Lack of improvement in vitamin A status with increased consumption of dark-green leafy vegetables. Lancet, v. 346, p. 7581, 1995.

[12] de PEE, S.; WEST, C. E.; PERMAESIH, D.; MARTUTI, S.; MUHILAL; HAUTVAST, J. G. A. Orange fruit is more effective than are dark-green, leafy vegetables in increasing serum concentrations of retinol and $\beta$ carotene in schoolchildren in Indonesia. Am. J. Clin. Nutr. v. 68, p. 10581067, 1998.

[13] EDWARDS, A. J.; NGUYEN, C. H.; YOU, C.; SWANSON, J. E.; EMENHISER, C.; PARKER, R. S. $\alpha$ and $\beta$-Carotene from a Commercial Carrot Puree Are More Bioavailable to Humans than from Boiled-Mashed Carrots, as Determined Using an Extrinsic Stable Isotope Reference Method. J. Nutr., v. 132, p. 159167, 2002.

[14] FRANCO, G. Nutrição - texto básico e tabela de composição quimica dos alimentos. $9^{\text {a }}$ ed. Rio de Janeiro, Atheneu, 1995. 307p.

[15] GERALDO, R. R. C.; PAIVA, S. A. R.; PITAS, A. M. C. S.; GODOY, I.; CAMPANA, A. O. Distribuição da hipovitaminose A no Brasil nas últimas quatro décadas: ingestão alimentar, sinais clínicos e dados bioquímicos. Rev. Nutr., v. 16, n. 4, p. 443-460, 2003.

[16] GODOY, H. \& RODRIGUEZ-AMAYA, D. B. Ocurrence of cis-isomers of provitamin A in Brasilian Fruits. J. Agric. Food Chem., v. 42, p. 1306-1313, 1994.

[17] GODOY, H. T. \& RODRIGUEZ-AMAYA, D. Occurrence of Cis Isomers of Provitamins A in Brazilian Vegetables. J. Agric. Food Chem., v. 46, p. 3081-3086, 1998.

[18] GRANADO, F.; OLMEDILLA, B.; BLANCO, I.; ROJASHIDALGO, E. Carotenoid Composition in Raw and
Cooked Spanish Vegetables. J. Agric. Food Chem., v. 40, p. 2135-2140. 1992.

[19] HART, D. J. \& SCOTT, K. J. Development and Evaluation of na HPLC Method for the Analysis of Carotenoids in Foods, and the Measurement of the Carotenoid Content of Vegetables and Fruits Commonly Consumed in the UK. Food Chem., v. 54, p. 101-111, 1995.

[20] IOM - U. S. Institute of Medicine, Food and Nutrition Board, Standing Committee on the Scientific Evaluation of Dietary Reference Intakes. Dietary Reference Intakes: for Vitamin A, Vitamin K, Arsenic, Boron, Cromium, Copper, Iodine, Iron, Manganese, Molybdenium, Nickel, Silicon, Vanadium and Zinc. Washington, D.C., National Academy Press, 2001, 797p.

[21] JALAL F, NESHEIM MC, AGUS Z, SANJUR D, HABICHT JP. Serum retinol concentrations in children are affected by food sources of $\beta$-carotene, fat intake, and anthelmintic drug treatment. Am. J. Clin. Nutr., v.68, p. 623629, 1998.

[22] KHACHIK, F; GOLI, M. B.; BEECHER, G. R.; HOLDEN, J.; LUSBY, W. R.; TENORIO, M. D.; BARRERA, M. R. Effect of Food Preparation on Qualitative and Quantitative Distribution of Major Carotenóid Constituents of Tomatoes and Several Green Vegetables. J. Agric. Food Chem., v. 40, p. 390-398, 1992.

[23] LESSin, W. J.; CATIGANI, G. L.; SCHWARTZ, S. J. Quantification of cis-trans Isomers of Provitamin A Carotenoids in Fresh and Processed Fruits and Vegetables. J. Agric. Food Chem., v.45, p. 3728-3732, 1997.

[24] MERCADANTE, A. Z.; RODRIGUEZ-AMAYA, D. B; BRITTON, G. HPLC and mass spectrometric analysis of carotenoids from mango. J. Agric. Food Chem., v. 45, p. 120-123, 1997.

[25] MICOZZI MS, BROWN ED, EDWARDS BK, et al. Plasma carotenoid response to chronic intake of selected foods and $\beta$-carotene supplements in men. Am. J. Clin. Nutr., v. 55, p.11201125, 1992.

[26] MOREIRA, A.P.B.; SANT'ANA, H.M.P.; SOUZA, S.L.; ALENCAR, E.R. Atividade Provitamíca A de Hortaliças Comercializadas no Mercado Formal e Informal de Viçosa, MG. Rev. Ceres (artigo no prelo).

[27] NATIONAL ACADEMY OF SCIENCE / NATIONAL COUNCIL RESEARCH (NAS-NCR). Recommended dietary allowances. $9^{a}$ ed., Washington, D.C., 1989. p. 78-92.

[28] NCUBE, T. N.; GREINER, T.; MALABA, L. C.; GEBREMEDHIN, M. Supplementing Lactating Women with Puréed Papaya and Grated Carrots Improved Vitamin A Status in a Placebo-Controlled Trial. J. Nutr., v. 131, p. 14971502, 2001.

[29] OLSON, J. A. In: MACHLIN, L. J., ed. Handbook of Vitamins. $2^{\text {a }}$ ed, New York: Marcel Dekker, 1991. p. 157.

[30] PAHO (Pan American Health Organization). Providing vitamin $A$ supplements through immunization and other health contacts for children 0-59 months and women up to 6 weeks postpartum - A guide for health workers. $2^{\text {a }}$ ed, 2001.35p. 
[31] PINHEIRO-SANT'ANA, H. M.; STRINGHETA, P. C.; BRANDÃO, S. C. C.; AZEREDO, R. M. C. Carotenoid retention and vitamin A value in carrot (Daucus carota L.) prepared by food service. Food Chem., v. 61, n. 1-2, p. 145-151, 1998.

[32] RIBAYA-MERCADO, J. D.; SOLON, F. S.; SOLON, M. A.; CABAL-BARZA, M. A.; PERFECTO, C. S.; TANG, G.; SOLON, J. A.; FJELD, C. R.; RUSSELL, R. M. Bioconversion of plant carotenoids to vitamin $\mathrm{A}$ in Filipino school-aged children varies inversely with vitamin A status. Am. J. Clin. Nutr., v. 72, p. 455465, 2000.

[33] ROCK, C. L.; LOVALVO, J.L.; EMENHISER, C.; RUFFIN, M.T.; FLATT, S. W. \& SCHWARTZ, S.J. Bioavailability of $\beta$-Carotene Is Lower In Raw than in Processed Carrots and Spinach in Women. J. Nutr., v. 128, p. 913-916, 1998.

[34] RODRIGUEZ-AMAYA, D. B. Critical review of provitamin A determination in plant foods. J. Micronutr. Anal., v. 5, p. 191-225, 1989.

[35] RODRIGUEZ-AMAYA, D.B. Nature and distribution of carotenoids in foods. In: Charalambous, F. (ed.). Shelf life of foods and beverages chemical, biological, physical and nutritional aspects. Amsterdam, Elsevier Science. 1993, p. 547-89.

[36] RODRIGUEZ-AMAYA, D.B. Some considerations in generating carotenoid data for food composition tables. J. Food Composition Anal., v. 13, p. 641-647, 2000.

[37] SÁ, M. C. \& RODRIGUEZ-AMAYA, D. B. Carotenoid composition of cooked green vegetables from restaurants. Food Chem., v. 83, p. 595600, 2003.

[38] SARNI, R.S.; KOCHI, C.; RAMALHO, R.A.; SCHOEPS, D.O.; SATO, K.; MATTOSO, L.C.Q.; XIMENES, C.F.; SOUZA, F.I.S. \& DAMIANI, F.M. Vitamina A: nivel sérico e ingestão dietética em crianças e adolecentes com déficit estatural de causa nãoo hormonal. Rev. Assoc. Med. Bras., v. 48, p. 48-53, 2002.

[39] SILVA, S. R. \& MERCADANTE, A. Z. Composição de carotenóides de maracujá-amarelo (Passiflora edulis flavicarpa) in natura. Ciênc. Tecnol. Aliment., v. 22, n. 3, p. 254-258, 2002.
[40] SOMMER, A. La carencia de vitamina A y sus consecuencias. Guía práctica para la detección y el tratamiento. $3^{\mathrm{a}}$ ed, Ginebra, Organización Mundial de la Salud, 1995.

[41] SOUZA, S. L., MOREIRA, A. P. B., PINHEIRO-SANT'ANA, H. M. Conteúdo de carotenos e provitamina A em frutas comercializadas em Viçosa, MG. Acta Scientiarum (artigo no prelo).

[42] TANG, G.; GU, X.; HU, S.; XU, Q.; QIN, J.; DOLNIKOWSKI, G. G.; FJELD, C. R.; GAO, X.; RUSSELL, R. M;YIN, S. Green and yellow vegetables can maintain body stores of vitamin A in Chinese children. Am. J. Clin. Nutr., v. 70, n. 6, p. 1069-1076. 1999.

[43] TORRONEN, R.; LEHMUSAHO, M.; HAKKINEN, S.; HANNINEN, O.; MYKKANEN, H. Serum $\beta$-carotene response to supplementation with raw carrots, carrot juice or purified b-carotene in healthy non-smoking women. Nutr. Res., v. 16, p. 565575, 1996.

[44] VAN het HOF, K. H.; BROUWER, I. A.; WEST, C. E.; HADDEMAN, E.; STEEGERS-THEUNISSEN, R. P. M.; VAN DUSSELDORP, M.; WESTSTRATE, J. A.; ESKES, T. K. A. B.; HAUTVAST, J. G. A. J. Bioavailability of lutein from vegetables is 5 times higher than that of $\beta$-carotene. Am. J. Clin. Nutr., v. 70, p. 261-268, 1999.

[45] VAN het HOF, K. H.; WEST, C. E.; WESTSTRATE, J. A.; HAUTVAST, J. G. A. J. Dietary Factors That Affect the Bioavailability of Carotenoids. J. Nutr., v. 130, p. 503506, 2000.

[46] VAN LIESHOUT, M.; WEST, C. E.; MUHILAL; PERMAESIH, D.; WANG, Y.; XU, X.; VAN BREEMEN, R. B.; CREEMERS, A. F. L.; VERHOEVEN, M. A.; LUGTENBURG, J. Bioefficacy of $\beta$-carotene dissolved in oil studied in children in Indonesia. Am. J. Clin. Nutr., v. 73, p. 949-958, 2001.

[47] WEST, C. E., EILANDER, A., VAN LIESHOUT, M. Consequences of Revised Estimates of Carotenoid Bioefficacy for Dietary Control of Vitamin A Deficiency in Developing Countries. J. Nutr., v. 132, p. 2920S2926S, 2002.

[48] WHO, Global prevalence of vitamin A deficiency. World Health Organization, Geneva, 1995, (WHO/NUT /95.3). 